\title{
Properties of solutions to fractional $p$-subLaplace equations on the Heisenberg group
}

\author{
Xinjing Wang ${ }^{1}$ and Guangwei $\mathrm{Du}^{2^{*}}$ (D)
}

\section{"Correspondence:}

guangwei87@mail.nwpu.edu.cn ${ }^{2}$ School of Mathematical Sciences, Qufu Normal University, Qufu, Shandong 273165, China Full list of author information is available at the end of the article

\section{Springer}

\begin{abstract}
The aim of this paper is to study properties of solutions to the fractional $p$-sublaplace equations on the Heisenberg group. Based on the maximum principles and the generalization of the direct method of moving planes, we obtain the symmetry and monotonicity of the solutions on the whole group and the Liouville property of solutions on a half space.
\end{abstract}

MSC: Primary 35A01; secondary 35J57; 35D99

Keywords: Fractional $p$-subLaplace equation; Heisenberg group; Maximum principle; Direct method of moving planes

\section{Introduction}

Let

$$
\left(-\Delta_{\mathbb{H}}\right)_{p}^{s} u(\xi)=C_{Q, s, p} P V \int_{\mathbb{H}^{n}} \frac{|u(\xi)-u(\eta)|^{p-2}(u(\xi)-u(\eta))}{\left|\eta^{-1} \circ \xi\right|_{\mathbb{H}}^{Q+s p}} d \eta
$$

be the fractional $p$-subLaplacian on the Heisenberg group $\mathbb{H}^{n}$, where $0<s<1, Q=2 n+2$, $C_{Q, s}$ is a positive constant, and $P V$ is the Cauchy principal value. In this paper we study the properties of cylindrical solutions to the fractional $p$-subLaplace equation

$$
\left(-\Delta_{\mathbb{H}}\right)_{p}^{s} u(\xi)=f(u(\xi))
$$

where $2 \leq p<\infty$.

Recall that the fractional Laplacian in $\mathbb{R}^{n}$ is a nonlocal pseudodifferential operator defined by

$$
(-\Delta)^{\alpha} u(x)=C_{n, \alpha} \lim _{\varepsilon \rightarrow 0} \int_{\mathbb{R}^{n} \backslash B_{\varepsilon}(x)} \frac{u(x)-u(y)}{|x-y|^{n+2 \alpha}} d y,
$$

where $0<\alpha<1, C_{n, \alpha}$ is a constant, and $u$ belongs to the Schwartz space. Since the nonlocal property of the operator $(-\Delta)^{\alpha}$ brings new difficulties to investigate, Caffarelli and

(c) The Author(s) 2020. This article is licensed under a Creative Commons Attribution 4.0 International License, which permits use, sharing, adaptation, distribution and reproduction in any medium or format, as long as you give appropriate credit to the original author(s) and the source, provide a link to the Creative Commons licence, and indicate if changes were made. The images or other third party material in this article are included in the article's Creative Commons licence, unless indicated otherwise in a credit line to the material. If material is not included in the article's Creative Commons licence and your intended use is not permitted by statutory regulation or exceeds the permitted use, you will need to obtain permission directly from the copyright holder. To view a copy of this licence, visit http://creativecommons.org/licenses/by/4.0/. 
Silvestre in [4] developed the extension method which can reduce the nonlocal problem relating to $(-\Delta)^{\alpha}$ to a local one in higher dimensions. This method has been applied to deal with equations involving the fractional Laplacian, and fruitful results have been obtained, see [3] and the references therein. Chen et al. [7] developed a direct method of moving planes to handle the problem involving $(-\Delta)^{\alpha}$ for $0<\alpha<1$, and this direct method has been used successfully to study symmetry, monotonicity, and nonexistence for many fractional Laplace equations, see [6, 7] and the references therein. Recently, Chen and Li [6] considered the fractional $p$-Laplacian

$$
(-\Delta)_{p}^{\alpha} u(x)=C_{n, \alpha, p} P V \int_{\mathbb{R}^{n}} \frac{|u(x)-u(y)|^{p-2}(u(x)-u(y))}{|x-y|^{n+\alpha p}} d y
$$

and obtained the radial symmetry and monotonicity of solutions to the equations involving operator (1.4).

To the elliptic equation

$$
-\Delta u=g(u)
$$

in $\mathbb{R}^{n}, \mathrm{Li}$ and $\mathrm{Ni}$ [19] proved that the positive solutions to (1.5) are radially symmetric with the assumptions that the limit of $u$ is zero at the infinity and $g^{\prime} \leq 0$ if $u$ is sufficiently small. Under the same conditions, the authors in [6] extended the result in [19] to the fractional $p$-Laplace equation

$$
(-\Delta)_{p}^{\alpha} u=g(u)
$$

and got the radial symmetry and monotonicity of the solutions. They also pointed out that the fractional $p$-Laplacian becomes $p$-Laplacian as $\alpha \rightarrow 1$ and, furthermore, it reduces to $-\Delta$ when $p=2$.

There are many interesting results about subLaplace and $p$-subLaplace equations on the Heisenberg group (see $[13,15,17,18]$ and $[10,11,20,21,25-27])$. There have been several different definitions of the fractional power subLaplacian in $\mathbb{H}^{n}$ (see $[12,14,22]$ etc.). The definition of fractional power subLaplacian given by Roncal and Thangavelu in [22] is indeed a generalization of the definition given by Cowling and Haagerup in [9] about the heat semigroup. The fractional power subLaplace equations can also be studied by generalizing the extension method in [4] to $\mathbb{H}^{n}$, although the fractional power subLaplacian $\left(-\Delta_{\mathbb{H}}\right)^{s}(0<s<1)$ does not have the concrete integral expression, for example, see [14] and [8] for $s=\frac{1}{2}$. There are also some results of the fractional power subLaplacian which are the extension of $[8]$, see $[23,24]$. Note that the expression of fractional power subLaplacian on $\mathbb{H}^{n}$ (see [22])

$$
\left(-\Delta_{\mathbb{H}}\right)^{s} u(\xi)=C_{Q, s} P V \int_{\mathbb{H}^{n}} \frac{u(\xi)-u(\eta)}{\left|\eta^{-1} \circ \xi\right|_{\mathbb{H}}{ }^{Q+2 s}} d \eta
$$

is the special form of fractional $p$-subLaplacian (1.1). By extending the method of moving planes in [5-7] to $\mathbb{H}^{n}$, in this paper, we study the properties of the solutions to (1.2) on $\mathbb{H}^{n}$ and $\mathbb{H}_{+}^{n}=\left\{\xi \in \mathbb{H}^{n} \mid t>0\right\}$.

Our main results are the following. 
Theorem 1.1 Let $0<s<1,2 \leq p<\infty$, and $u \in L_{s p}\left(\mathbb{H}^{n}\right) \cap C_{\text {loc }}^{1,1}\left(\mathbb{H}^{n}\right)$ be a nonnegative cylindrical solution to (1.2) with

$$
\lim _{|\xi| \mathbb{H} \rightarrow \infty} u(\xi)=0,
$$

and suppose that $f^{\prime}(a)$ is nonpositive and locally bounded for a sufficiently small. Then $u$ must be symmetric and monotone with respect to t about some point in $\mathbb{H}^{n}$.

Theorem 1.2 Let $0<s<1,2 \leq p<\infty$, and $u \in L_{s p}\left(\mathbb{H}_{+}^{n}\right) \cap C_{\mathrm{loc}}^{1,1}\left(\mathbb{H}_{+}^{n}\right)$ be a nonnegative cylindrical solution to the problem

$$
\left\{\begin{array}{l}
\left(-\Delta_{\mathbb{H}}\right)_{p}^{s} u(\xi)=f(u(\xi)), \quad \xi \in \mathbb{H}_{+}^{n}, \\
u(\xi)=0, \quad \xi \notin \mathbb{H}_{+}^{n},
\end{array}\right.
$$

and suppose that $u$ satisfies (1.8) and is lower semicontinuous on $\overline{\mathbb{H}}_{+}^{n}$. If $f(0)=0, f^{\prime}(a)$ is nonpositive and locally bounded for a sufficiently small, then $u \equiv 0$.

Observe that Theorem 1.1 is the extension of symmetry and monotonicity of solutions to the fractional $p$-Laplace equation on $\mathbb{R}^{n}$ in [6] to the Heisenberg group, and Theorem 1.2 is the Liouville property on a half space in $\mathbb{H}^{n}$. When $f(a)=-a+a^{q}(q>1)$, our results still hold.

The authors in [22] assumed that $u \in C_{0}^{\infty}\left(\mathbb{H}^{n}\right)$ in (1.7). We point out that (1.1) is also well defined for $u \in L_{s p}\left(\mathbb{H}^{n}\right) \cap C_{\text {loc }}^{1,1}\left(\mathbb{H}^{n}\right)$, where

$$
L_{s p}\left(\mathbb{H}^{n}\right)=\left\{u: \mathbb{H}^{n} \rightarrow \mathbb{R} \mid \int_{\mathbb{H}^{n}} \frac{|u(\xi)|^{p-1}}{1+|\xi|_{\mathbb{H}}^{Q+s p}} d \xi<\infty\right\} .
$$

The paper is organized as follows. Section 2 collects some well-known results on $\mathbb{H}^{n}$, and we show that (1.1) is well defined for $u \in L_{s p}\left(\mathbb{H}^{n}\right) \cap C_{\text {loc }}^{1,1}\left(\mathbb{H}^{n}\right)$. In Sect. 3, we establish three maximum principles. Theorem 1.1 and Theorem 1.2 are proved in Sect. 4.

\section{Preliminaries}

The Heisenberg group $\mathbb{H}^{n}$ is the Euclidean space $\mathbb{R}^{2 n+1}(n \geq 1)$ endowed with the group law o:

$$
\bar{\xi} \circ \xi=\left(x+\bar{x}, y+\bar{y}, t+\bar{t}+2 \sum_{i=1}^{n}\left(x_{i} \bar{y}_{i}-y_{i} \bar{x}_{i}\right)\right),
$$

where $\xi=\left(x_{1}, \ldots, x_{n}, y_{1}, \ldots, y_{n}, t\right):=(x, y, t) \in \mathbb{R}^{n} \times \mathbb{R}^{n} \times \mathbb{R}$ and $\bar{\xi}=(\bar{x}, \bar{y}, \bar{t})$. Denote by $\delta_{\kappa}$ the dilations on $\mathbb{H}^{n}$, i.e.,

$$
\delta_{\kappa}(\xi)=\left(\kappa x, \kappa y, \kappa^{2} t\right), \quad \kappa>0,
$$

which satisfy $\delta_{\kappa}(\bar{\xi} \circ \xi)=\delta_{\kappa}(\bar{\xi}) \circ \delta_{\kappa}(\xi)$.

The left invariant vector fields corresponding to $\mathbb{H}^{n}$ are

$$
X_{i}=\frac{\partial}{\partial x_{i}}+2 y_{i} \frac{\partial}{\partial t}, \quad i=1, \ldots, n,
$$




$$
\begin{aligned}
Y_{i} & =\frac{\partial}{\partial y_{i}}-2 x_{i} \frac{\partial}{\partial t}, \quad i=1, \ldots, n, \\
T & =\frac{\partial}{\partial t} .
\end{aligned}
$$

It is easy to check that $X_{i}$ and $Y_{j}$ satisfy

$$
\left[X_{i}, Y_{j}\right]=-4 T \delta_{i j}, \quad\left[X_{i}, X_{j}\right]=\left[Y_{i}, Y_{j}\right]=0, \quad i, j=1, \ldots, n
$$

The Heisenberg gradient of a function $u$ is defined by

$$
\nabla_{\mathbb{H}} u=\left(X_{1} u, \ldots, X_{n} u, Y_{1} u, \ldots, Y_{n} u\right)
$$

and the subLaplacian $\Delta_{\mathbb{H}}$ on $\mathbb{H}^{n}$ is

$$
\begin{aligned}
\Delta_{\mathbb{H}} & :=\sum_{i=1}^{n}\left(X_{i}^{2}+Y_{i}^{2}\right) \\
& =\sum_{i=1}^{n}\left(\frac{\partial^{2}}{\partial x_{i}^{2}}+\frac{\partial^{2}}{\partial y_{i}^{2}}+4 y_{i} \frac{\partial^{2}}{\partial x_{i} \partial t}-4 x_{i} \frac{\partial^{2}}{\partial y_{i} \partial t}+4\left(x_{i}^{2}+y_{i}^{2}\right) \frac{\partial^{2}}{\partial t^{2}}\right) .
\end{aligned}
$$

The family $\left\{X_{1}, \ldots, X_{n}, Y_{1}, \ldots, Y_{n}\right\}$ satisfies Hörmander's rank condition (see [16]) which implies that $\Delta_{\mathbb{H}}$ is hypoelliptic and the maximum principle holds for solutions to the equation involving $\Delta_{\mathbb{H}}($ see [2]).

The integer $Q=2 n+2$ is called the homogeneous dimension of $\mathbb{H}^{n}$. Denote by $|\xi|_{\mathbb{H}}$ the distance from $\xi$ to the zero (see [13])

$$
|\xi|_{\mathbb{H}}=\left(\sum_{i=1}^{n}\left(x_{i}^{2}+y_{i}^{2}\right)^{2}+t^{2}\right)^{\frac{1}{4}}
$$

Authors in [22] used the norm $|(z, w)|=\left(\sum_{i=1}^{n}\left(x_{i}{ }^{2}+y_{i}{ }^{2}\right)^{2}+16 t^{2}\right)^{\frac{1}{4}}$ for $(x, y, t):=(z, w) \in \mathbb{H}^{n}$, which is equivalent to (2.5). The distance between two points of $\mathbb{H}^{n}$ is defined by

$$
d_{\mathbb{H}}(\xi, \eta)=\left|\eta^{-1} \circ \xi\right|_{\mathbb{H}}
$$

where $\eta^{-1}$ denotes the inverse of $\eta$ with respect to $\circ$, that is, $\eta^{-1}=-\eta$. The open ball of radius $R>0$ centered at $\xi$ is the set

$$
B_{\mathbb{H}}(\xi, R)=\left\{\eta \in \mathbb{H}^{n} \mid d_{\mathbb{H}}(\eta, \xi)<R\right\} .
$$

It is well known that $\xi \rightarrow|\xi|_{\mathbb{H}}$ is homogeneous of degree one with respect to $\delta_{\kappa}$ and

$$
\left|B_{\mathbb{H}}(\xi, R)\right|=\left|B_{\mathbb{H}}(0, R)\right|=\left|B_{\mathbb{H}}(0,1)\right| R^{Q},
$$

where $|\cdot|$ denotes the Lebesgue measure.

A function $u$ is called the cylindrical function if

$$
u(x, y, t)=u(r, t)
$$


where $(x, y, t) \in \mathbb{H}^{n}, r=\left(|x|^{2}+|y|^{2}\right)^{\frac{1}{2}}$.

Proposition 2.1 For $u \in L_{s p}\left(\mathbb{H}^{n}\right) \cap C_{\mathrm{loc}}^{1,1}\left(\mathbb{H}^{n}\right)$, the operator in (1.1) is well defined.

Proof For any $\xi \in \mathbb{H}^{n}$,

$$
\begin{aligned}
P V & \int_{\mathbb{H}^{n}} \frac{|u(\xi)-u(\eta)|^{p-2}(u(\xi)-u(\eta))}{\left|\eta^{-1} \circ \xi\right|_{\mathbb{H}}^{Q+s p}} d \eta \\
= & \lim _{\varepsilon \rightarrow 0}\left[\int _ { B _ { \mathbb { H } } ( \xi , 1 ) \backslash B _ { \mathbb { H } } ( \xi , \varepsilon ) } \left(\left|\left\langle-\left(\nabla_{\mathbb{H}} u, T u\right), \eta^{-1} \circ \xi\right\rangle+o\left(\left|\eta^{-1} \circ \xi\right|_{\mathbb{H}}^{2}\right)\right|^{p-2}\right.\right. \\
& \left.\times\left(\left|-\left(\nabla_{\mathbb{H}} u, T u\right), \eta^{-1} \circ \xi\right\rangle+o\left(\left|\eta^{-1} \circ \xi\right|_{\mathbb{H}}^{2}\right)\right)\right)\left(\left|\eta^{-1} \circ \xi\right|_{\mathbb{H}}^{Q+s p}\right)^{-1} d \eta \\
& \left.+\int_{\mathbb{H}^{n} \backslash B_{\mathbb{H}}(\xi, 1)} \frac{|u(\xi)-u(\eta)|^{p-2}(u(\xi)-u(\eta))}{\left|\eta^{-1} \circ \xi\right|_{\mathbb{H}}^{Q+s p}} d \eta\right] \\
\leq & C \lim _{\varepsilon \rightarrow 0}\left[\int_{B_{\mathbb{H}}(\xi, 1) \backslash B_{\mathbb{H}}(\xi, \varepsilon)} \frac{o(1)}{\left|\eta^{-1} \circ \xi\right|_{\mathbb{H}}^{Q+s p}} d \eta+\int_{B_{\mathbb{H}}(\xi, 1) \backslash B_{\mathbb{H}}(\xi, s)} \frac{o(1)}{\left|\eta^{-1} \circ \xi\right|_{\mathbb{H}}^{Q+s p}} d \eta\right. \\
& \left.+\int_{\mathbb{H}^{n} \backslash B_{\mathbb{H}}(\xi, 1)} \frac{u^{p-1}(\xi)}{\left|\eta^{-1} \circ \xi\right|_{\mathbb{H}}^{Q+s p}} d \eta+\int_{\mathbb{H}^{n} \backslash B_{\mathbb{H}}(\xi, 1)} \frac{u^{p-1}(\eta)}{\left|\eta^{-1} \circ \xi\right|_{\mathbb{H}}^{Q+s p}} d \eta\right] \\
:= & C \lim _{\varepsilon \rightarrow 0}\left(I_{1}+I_{2}+I_{3}+I_{4}\right),
\end{aligned}
$$

where $\varepsilon$ is sufficiently small. Noting that $u \in C_{\text {loc }}^{1,1}\left(\mathbb{H}^{n}\right), Q+s p-p<Q$, and $Q+s p-2 p+2<Q$, we know that $I_{1}$ and $I_{2}$ are finite; $I_{3}$ is clearly convergent when $|\xi|_{\mathbb{H}} \rightarrow \infty$; and $I_{4}$ is finite from $u \in L_{s p}\left(\mathbb{H}^{n}\right)$. Hence, (1.1) is well defined.

\section{Maximum principles}

In this section, we prove three maximum principles which will be used in the process of moving planes. These maximum principles are on a bounded domain in $\mathbb{H}^{n}$, on a bounded domain in the left domain of some hyperplane, and on a narrow region.

Lemma 3.1 Let $\Omega$ be a bounded domain in $\mathbb{H}^{n}$. Assume $u \in L_{s p}\left(\mathbb{H}^{n}\right) \cap C_{\text {loc }}^{1,1}\left(\mathbb{H}^{n}\right)$ is lower semicontinuous on $\bar{\Omega}$ and satisfies

$$
\left\{\begin{array}{l}
\left(-\Delta_{\mathbb{H}}\right)_{p}^{s} u(\xi) \geq 0, \quad \xi \in \Omega, \\
u(\xi) \geq 0, \quad \xi \in \mathbb{H}^{n} \backslash \Omega,
\end{array}\right.
$$

then

$$
u(\xi) \geq 0, \quad \xi \in \Omega .
$$

Furthermore, if $u=0$ at some point in $\Omega$, then

$$
u(\xi)=0 \quad \text { almost everywhere in } \mathbb{H}^{n} .
$$

These conclusions also hold on the unbounded region $\Omega$ if we further assume that

$$
\varliminf_{|\xi| \mathbb{\mathbb { H }} \rightarrow \infty} u(\xi) \geq 0 .
$$


Proof Suppose that (3.2) is not true, then by the lower semicontinuity of $u$ on $\bar{\Omega}$ there exists $\xi^{0} \in \bar{\Omega}$ such that

$$
u\left(\xi^{0}\right)=\min _{\bar{\Omega}} u<0
$$

From (3.1), we know that $\xi^{0}$ is a point in $\Omega$, and

$$
\begin{aligned}
\left(-\Delta_{\mathbb{H}}\right)_{p}^{s} u\left(\xi^{0}\right) & =C_{Q, s, p} P V \int_{\mathbb{H}^{n}} \frac{\left|u\left(\xi^{0}\right)-u(\eta)\right|^{p-2}\left(u\left(\xi^{0}\right)-u(\eta)\right)}{\left|\eta^{-1} \circ \xi^{0}\right|_{\mathbb{H}^{Q}}{ }^{+s p}} d \eta \\
& \leq C_{Q, s, p} \int_{\mathbb{H}^{n} \backslash \Omega} \frac{\left|u\left(\xi^{0}\right)-u(\eta)\right|^{p-2}\left(u\left(\xi^{0}\right)-u(\eta)\right)}{\left|\eta^{-1} \circ \xi^{0}\right|_{\mathbb{H}^{Q}}^{Q+s p}} d \eta \\
& <0,
\end{aligned}
$$

which contradicts (3.1). This implies (3.2).

If there exists some point $\xi^{0} \in \Omega$ such that $u\left(\xi^{0}\right)=0$, then

$$
0 \leq\left(-\Delta_{\mathbb{H}}\right)_{p}^{s} u\left(\xi^{0}\right)=C_{Q, s, p} \int_{\mathbb{H}^{n}} \frac{|u(\eta)|^{p-2}(-u(\eta))}{\left|\eta^{-1} \circ \xi^{0}\right|_{\mathbb{H}}^{Q+s p}} d \eta
$$

Using $u(\xi) \geq 0$, we have $u(\xi)=0$ almost everywhere in $\mathbb{H}^{n}$.

For an unbounded region $\Omega$, the condition $\underline{\lim }_{|\xi|_{\mathbb{H}} \rightarrow \infty} u(\xi) \geq 0$ implies that the negative minimum $\xi^{0}$ of $u$ cannot be reached at infinity. Then the condition of lower semicontinuity ensures that the proof can go on as above. The proof is ended.

Let $T_{\lambda}$ be a hyperplane in $\mathbb{H}^{n}$ defined by

$$
T_{\lambda}=\left\{\xi \in \mathbb{H}^{n} \mid t=\lambda, \lambda \in \mathbb{R}\right\} .
$$

Denote by $\tilde{\xi}=(y, x, 2 \lambda-t)$ the H-reflection of $\xi=(x, y, t)$ about the plane $T_{\lambda}$ and by

$$
\Sigma_{\lambda}=\left\{\xi \in \mathbb{H}^{n} \mid t<\lambda\right\}
$$

the region in the left of the plane $T_{\lambda}$. Letting

$$
u_{\lambda}(\xi)=u_{\lambda}(|(x, y)|, t):=u(|(x, y)|, 2 \lambda-t)
$$

and using the $\mathrm{H}$-refection (see [1]), we have

$$
u_{\lambda}(\xi)=u(y, x, 2 \lambda-t)=u\left(\xi^{\lambda}\right)
$$

Set

$$
w_{\lambda}(\xi)=u_{\lambda}(\xi)-u(\xi) .
$$


Lemma 3.2 Let $\Omega$ be a bounded domain in $\Sigma_{\lambda}$. Assume that the cylindrical function $u \in$ $L_{s p}\left(\mathbb{H}^{n}\right) \cap C_{\text {loc }}^{1,1}\left(\mathbb{H}^{n}\right)$ is lower semicontinuous on $\bar{\Omega}$ and satisfies

$$
\left\{\begin{array}{l}
\left(-\Delta_{\mathbb{H}}\right)_{p}^{s} u_{\lambda}(\xi)-\left(-\Delta_{\mathbb{H}}\right)_{p}^{s} u(\xi) \geq 0, \quad \xi \in \Omega \\
w_{\lambda}(\xi) \geq 0, \quad \xi \in \Sigma_{\lambda} \backslash \Omega \\
w_{\lambda}\left(\xi^{\lambda}\right)=-w_{\lambda}(\xi), \quad \xi \in \Sigma_{\lambda}
\end{array}\right.
$$

then

$$
w_{\lambda}(\xi) \geq 0, \quad \xi \in \Omega
$$

Furthermore, if $w_{\lambda}=0$ at some point in $\Omega$, then

$$
w_{\lambda}(\xi)=0 \quad \text { almost everywhere in } \mathbb{H}^{n} .
$$

These conclusions also hold for the unbounded region $\Omega$ if we further assume that

$$
\varliminf_{|\xi| \mathbb{H} \rightarrow \infty} w_{\lambda}(\xi) \geq 0
$$

Proof Suppose that (3.4) is incorrect. By the lower semicontinuity of $w_{\lambda}$ on $\bar{\Omega}$, there exists $\xi^{0} \in \bar{\Omega}$ such that

$$
w_{\lambda}\left(\xi^{0}\right)=\min _{\bar{\Omega}} w_{\lambda}<0
$$

For simplicity, we denote

$$
G(a)=|a|^{p-2} a, \quad a \geq 0 .
$$

Note that $G(a)$ is increasing and $G^{\prime}(a)=(p-1)|a|^{p-2} \geq 0$. A direct calculation gives

$$
\begin{aligned}
\left(-\Delta_{\mathbb{H}}\right)_{p}^{s} u_{\lambda}\left(\xi^{0}\right)-\left(-\Delta_{\mathbb{H}}\right)_{p}^{s} u\left(\xi^{0}\right) \\
=C_{Q, s, p} P V \int_{\mathbb{H}^{n}} \frac{G\left(u_{\lambda}\left(\xi^{0}\right)-u_{\lambda}(\eta)\right)-G\left(u\left(\xi^{0}\right)-u(\eta)\right)}{\left|\eta^{-1} \circ \xi^{0}\right|_{\mathbb{H}}{ }^{Q+s p}} d \eta \\
=C_{Q, s, p} P V \int_{\Sigma_{\lambda}} \frac{G\left(u_{\lambda}\left(\xi^{0}\right)-u_{\lambda}(\eta)\right)-G\left(u\left(\xi^{0}\right)-u(\eta)\right)}{\left|\eta^{-1} \circ \xi^{0}\right|_{\mathbb{H}}^{Q+s p}} d \eta \\
\quad+C_{Q, s, p} P V \int_{\Sigma_{\lambda}} \frac{\left.G\left(u_{\lambda} \xi^{0}\right)-u(\eta)\right)-G\left(u\left(\xi^{0}\right)-u_{\lambda}(\eta)\right)}{\left|\left(\eta^{\lambda}\right)^{-1} \circ \xi^{0}\right|_{\mathbb{H}}^{Q+s p}} d \eta \\
=C_{Q, s, p} P V \int_{\Sigma_{\lambda}}\left(\frac{1}{\left|\eta^{-1} \circ \xi^{0}\right|_{\mathbb{H}}{ }^{Q+s p}}-\frac{1}{\left|\left(\eta^{\lambda}\right)^{-1} \circ \xi^{0}\right|_{\mathbb{H}}{ }^{Q+s p}}\right) \\
\quad \times\left(G\left(u_{\lambda}\left(\xi^{0}\right)-u_{\lambda}(\eta)\right)-G\left(u\left(\xi^{0}\right)-u(\eta)\right)\right) d \eta \\
\quad+C_{Q, s, p} P V \int_{\Sigma_{\lambda}}\left(G\left(u_{\lambda}\left(\xi^{0}\right)-u_{\lambda}(\eta)\right)-G\left(u\left(\xi^{0}\right)-u_{\lambda}(\eta)\right)+G\left(u_{\lambda}\left(\xi^{0}\right)-u(\eta)\right)\right.
\end{aligned}
$$




$$
\begin{aligned}
& \left.-G\left(u\left(\xi^{0}\right)-u(\eta)\right)\right)\left(\left|\left(\eta^{\lambda}\right)^{-1} \circ \xi^{0}\right|_{\mathbb{H}}^{Q+s p}\right)^{-1} d \eta \\
:= & C_{Q, s, p}\left(J_{1}+J_{2}\right) .
\end{aligned}
$$

For $J_{1}$, we have for any $\xi^{0}, \eta \in \Sigma_{\lambda}$,

$$
\frac{1}{\left|\eta^{-1} \circ \xi^{0}\right|_{\mathbb{H}}^{Q+s p}}-\frac{1}{\left|\left(\eta^{\lambda}\right)^{-1} \circ \xi^{0}\right|_{\mathbb{H}}^{Q+s p}}>0 .
$$

By the monotonicity of $G$ and the fact that

$$
\left(u_{\lambda}\left(\xi^{0}\right)-u_{\lambda}(\eta)\right)-\left(u\left(\xi^{0}\right)-u(\eta)\right)=w_{\lambda}\left(\xi^{0}\right)-w_{\lambda}(\eta)
$$

is nonpositive but not identity to 0 , we deduce that

$$
G\left(u_{\lambda}\left(\xi^{0}\right)-u_{\lambda}(\eta)\right)-G\left(u\left(\xi^{0}\right)-u(\eta)\right)
$$

is also nonpositive but not identity to 0 . So we have

$$
J_{1}<0
$$

For $J_{2}$, by the mean value theorem,

$$
\begin{aligned}
J_{2} & =\int_{\Sigma_{\lambda}} \frac{G\left(u_{\lambda}\left(\xi^{0}\right)-u_{\lambda}(\eta)\right)-G\left(u\left(\xi^{0}\right)-u_{\lambda}(\eta)\right)+G\left(u_{\lambda}\left(\xi^{0}\right)-u(\eta)\right)-G\left(u\left(\xi^{0}\right)-u(\eta)\right)}{\left|\left(\eta^{\lambda}\right)^{-1} \circ \xi^{0}\right|_{\mathbb{H}}^{Q+s p}} d \eta \\
& =w_{\lambda}\left(\xi^{0}\right) \int_{\Sigma_{\lambda}} \frac{G^{\prime}(g(\eta))+G^{\prime}(h(\eta))}{\left|\left(\eta^{\lambda}\right)^{-1} \circ \xi^{0}\right|_{\mathbb{H}}^{Q+s p}} d \eta \\
& \leq 0 .
\end{aligned}
$$

In fact, if $u_{\lambda}(\eta) \geq u(\eta)$, then we have $w_{\lambda}(\eta) \geq 0$, i.e., (3.4) holds. If $u_{\lambda}(\eta)>u(\eta)$, we know $G$ is strictly increasing, then $G^{\prime}(g(\eta)) \geq 0$ and $G^{\prime}(h(\eta)) \geq 0$. Hence, we have (3.7).

Putting (3.6) and (3.7) into (3.5) implies

$$
\left(-\Delta_{\mathbb{H}}\right)_{p}^{s} u_{\lambda}\left(\xi^{0}\right)-\left(-\Delta_{\mathbb{H}}\right)_{p}^{s} u\left(\xi^{0}\right)<0
$$

This contradicts (3.3) and we obtain (3.4).

If there exists some point $\xi^{0} \in \Omega$ such that $w_{\lambda}\left(\xi^{0}\right)=0$, then (3.5) holds and $J_{2} \geq 0$. Hence from the first inequality in (3.3) we have $J_{1} \geq 0$, and by the monotonicity of $G$,

$$
G\left(u_{\lambda}\left(\xi^{0}\right)-u_{\lambda}(\eta)\right)-G\left(u\left(\xi^{0}\right)-u(\eta)\right) \geq 0
$$

We have, for almost all $\eta \in \Sigma_{\lambda}$,

$$
\left(u_{\lambda}\left(\xi^{0}\right)-u_{\lambda}(\eta)\right)-\left(u\left(\xi^{0}\right)-u(\eta)\right)=w_{\lambda}\left(\xi^{0}\right)-w_{\lambda}(\eta)=-w_{\lambda}(\eta) \geq 0 .
$$

Using (3.4), we have

$$
w_{\lambda}(\xi)=0 \quad \text { almost everywhere in } \Sigma_{\lambda} .
$$


From the antisymmetry of $w_{\lambda}$,

$$
w_{\lambda}(\xi)=0 \quad \text { almost everywhere in } \mathbb{H}^{n} .
$$

Lemma 3.3 Let $\Omega$ be a bounded narrow domain in $\Sigma_{\lambda}$ and locate in $\{\xi \mid \lambda-l<t<\lambda\}$ for small l. Assume that the cylindrical function $u \in L_{s p}\left(\mathbb{H}^{n}\right) \cap C_{\mathrm{loc}}^{1,1}\left(\mathbb{H}^{n}\right)$ is lower semicontinuous on $\bar{\Omega}$. If $c(x)$ is bounded from below in $\Omega$ and $u$ satisfies

$$
\left\{\begin{array}{l}
\left(-\Delta_{\mathbb{H}}\right)_{p}^{s} u_{\lambda}(\xi)-\left(-\Delta_{\mathbb{H}}\right)_{p}^{s} u(\xi)+c(\xi) w_{\lambda}(\xi) \geq 0, \quad \xi \in \Omega, \\
w_{\lambda}(\xi) \geq 0, \quad \xi \in \Sigma_{\lambda} \backslash \Omega \\
w_{\lambda}\left(\xi^{\lambda}\right)=-w_{\lambda}(\xi), \quad \xi \in \Sigma_{\lambda}
\end{array}\right.
$$

then

$$
w_{\lambda}(\xi) \geq 0, \quad \xi \in \Omega
$$

Furthermore, if $w_{\lambda}=0$ at some point in $\Omega$, then

$$
w_{\lambda}(\xi)=0 \quad \text { almost everywhere in } \mathbb{H}^{n} .
$$

These conclusions also hold for the unbounded region $\Omega$ if we further assume that

$$
\varliminf_{|\xi| \mathbb{H} \rightarrow \infty} w_{\lambda}(\xi) \geq 0
$$

Proof By the proof of Lemma 3.2, we have

$$
\begin{aligned}
&\left(-\Delta_{\mathbb{H}}\right)_{p}^{s} u_{\lambda}\left(\xi^{0}\right)-\left(-\Delta_{\mathbb{H}}\right)_{p}^{s} u\left(\xi^{0}\right) \\
&=C_{Q, s, p} P V \int_{\Sigma_{\lambda}}\left(\frac{1}{\left|\eta^{-1} \circ \xi^{0}\right|_{\mathbb{H}}^{Q+s p}}-\frac{1}{\left|\left(\eta^{\lambda}\right)^{-1} \circ \xi^{0}\right|_{\mathbb{H}}^{Q+s p}}\right) \\
& \quad \times\left(G\left(u_{\lambda}\left(\xi^{0}\right)-u_{\lambda}(\eta)\right)-G\left(u\left(\xi^{0}\right)-u(\eta)\right)\right) d \eta \\
& \quad+C_{Q, s, p} P V \int_{\Sigma_{\lambda}}\left(G\left(u_{\lambda}\left(\xi^{0}\right)-u_{\lambda}(\eta)\right)-G\left(u\left(\xi^{0}\right)-u(\eta)\right)+G\left(u_{\lambda}\left(\xi^{0}\right)-u(\eta)\right)\right. \\
& \\
&\left.\quad-G\left(u\left(\xi^{0}\right)-u_{\lambda}(\eta)\right)\right)\left(\left|\left(\eta^{\lambda}\right)^{-1} \circ \xi^{0}\right|_{\mathbb{H}}^{Q+s p}\right)^{-1} d \eta \\
&:= C_{Q, s, p}\left(I_{1}+I_{2}\right) .
\end{aligned}
$$

Obviously,

$$
I_{2} \leq 0
$$

Similar to (3.6), we know

$$
I_{1}<0 \text {. }
$$


Denote

$$
\delta_{\xi^{0}}=\operatorname{dist}\left(\xi^{0}, \partial \Sigma_{\lambda}\right)=\left|\lambda-t^{0}\right| .
$$

Combining (3.10), (3.11), and $I_{1}<0$, we have

$$
\frac{\left(-\Delta_{\mathbb{H}}\right)_{p}^{s} u_{\lambda}\left(\xi^{0}\right)-\left(-\Delta_{\mathbb{H}}\right)_{p}^{s} u\left(\xi^{0}\right)}{\delta_{\xi^{0}}}<0 .
$$

Noting that $\xi^{0}$ is a negative minimum of $w_{\lambda}$, we infer $\nabla w_{\lambda_{0}}\left(\xi^{0}\right)=0$, and so

$$
\frac{\partial w_{\lambda}}{\partial t}\left(\xi^{0}\right)=\lim _{\delta_{k} \rightarrow 0} \frac{w_{\lambda}\left(\xi^{0}\right)}{\delta_{\xi^{0}}}=0,
$$

i.e.,

$$
\frac{c\left(\xi^{0}\right) w_{\lambda}\left(\xi^{0}\right)}{\delta_{\xi^{0}}} \leq o(1)
$$

Now (3.12) and (3.13) contradict (3.8), and then (3.9) is proved.

\section{Proof of the main results}

Following the idea in [6], we first use Lemma 3.1, Lemma 3.2, and Lemma 3.3 to prove Theorem 1.1.

Proof of Theorem 1.1 First we check that for $\lambda$ sufficiently negative it holds

$$
w_{\lambda}(\xi) \geq 0, \quad \xi \in \Sigma_{\lambda} .
$$

Indeed, suppose that (4.1) is violated, then by (1.8) there exists a point $\xi^{0} \in \Sigma_{\lambda}$ such that

$$
w_{\lambda}\left(\xi^{0}\right)=\min _{\Sigma_{\lambda}} w_{\lambda}<0
$$

i.e., $u_{\lambda}\left(\xi^{0}\right) \leq \varsigma_{\lambda}\left(\xi^{0}\right) \leq u\left(\xi^{0}\right)$. Note by (1.2) that

$$
\left(-\Delta_{\mathbb{H}}\right)_{p}^{s} u_{\lambda}(\xi)-\left(-\Delta_{\mathbb{H}}\right)_{p}^{s} u(\xi)=f^{\prime}\left(\varsigma_{\lambda}(\xi)\right) w_{\lambda}(\xi)
$$

where $\varsigma_{\lambda}(\xi)$ between $u_{\lambda}(\xi)$ and $u(\xi)$. For sufficiently negative $\lambda, u\left(\xi^{0}\right)$ is small by (1.8), hence so is $\varsigma_{\lambda}\left(\xi^{0}\right)$. Due to the condition of $f^{\prime}$, we have $f^{\prime}\left(\varsigma_{\lambda}\left(\xi^{0}\right)\right) \leq 0$. From (4.2),

$$
\left(-\Delta_{\mathbb{H}}\right)_{p}^{s} u_{\lambda}\left(\xi^{0}\right)-\left(-\Delta_{\mathbb{H}}\right)_{p}^{s} u\left(\xi^{0}\right) \geq 0
$$

On the other hand, it follows by the proof of Lemma 3.2 that

$$
\left(-\Delta_{\mathbb{H}}\right)_{p}^{s} u_{\lambda}\left(\xi^{0}\right)-\left(-\Delta_{\mathbb{H}}\right)_{p}^{s} u\left(\xi^{0}\right)<0
$$

This contradicts (4.3), and hence (4.1) is proved. 
The above result provides the starting point of moving planes. Let us move the plane $T_{\lambda}$ to the right as long as (4.1) holds to its limiting position

$$
\lambda_{0}=\sup \left\{\lambda \mid w_{\mu}(\xi) \geq 0, \forall \xi \in \Sigma_{\mu}, \mu \leq \lambda\right\} .
$$

We will show that

$$
\lambda_{0}=0
$$

i.e.,

$$
w_{\lambda_{0}}(\xi)=0, \quad \xi \in \Sigma_{\lambda_{0}} .
$$

In fact, suppose that (4.6) is false, we have by Lemma 3.2 that

$$
w_{\lambda_{0}}(\xi)>0, \quad \xi \in \Sigma_{\lambda_{0}} .
$$

From the definition of $\lambda_{0}$, there exist a sequence $\lambda_{k} \rightarrow \lambda_{0}$ and a point $\xi^{k} \in \Sigma_{\lambda_{k}}$ such that

$$
w_{\lambda_{k}}\left(\xi^{k}\right)=\min _{\Sigma_{\lambda_{k}}} w_{\lambda_{k}}<0, \quad \nabla w_{\lambda_{k}}\left(\xi^{k}\right)=0
$$

Note that

$$
\left(-\Delta_{\mathbb{H}}\right)_{p}^{s} u_{\lambda_{k}}\left(\xi^{k}\right)-\left(-\Delta_{\mathbb{H}}\right)_{p}^{s} u\left(\xi^{k}\right)=f^{\prime}\left(\varsigma_{\lambda_{k}}\left(\xi^{k}\right)\right) w_{\lambda_{k}}\left(\xi^{k}\right)
$$

If $\left|\xi^{k}\right|_{\mathbb{H}}$ is sufficiently large, then $u\left(\xi^{k}\right)$ is small and so $\varsigma_{\lambda_{k}}\left(\xi^{k}\right)$ is also small, this implies $f^{\prime}\left(\varsigma_{\lambda_{k}}\left(\xi^{k}\right)\right) \leq 0$ (because $f^{\prime}(a) \leq 0$ for the sufficiently small $a$ ). It follows

$$
\left(-\Delta_{\mathbb{H}}\right)_{p}^{s} u_{\lambda_{k}}\left(\xi^{k}\right)-\left(-\Delta_{\mathbb{H}}\right)_{p}^{s} u\left(\xi^{k}\right) \geq 0
$$

But this contradicts the fact that $\xi^{k}$ is a negative minimum of $w_{\lambda_{k}}$ (see Lemma 3.2). Hence, $\left\{\xi^{k}\right\}$ is bounded, i.e., the sequence $\left\{\xi^{k}\right\}$ is bounded.

It follows that the subsequence of $\left\{\xi^{k}\right\}$ converges to some point $\xi^{0}$. Then (4.8) means that, for $\xi^{0} \in \partial \Sigma_{\lambda_{0}}$,

$$
w_{\lambda_{0}}\left(\xi^{0}\right) \leq 0, \quad \nabla w_{\lambda_{0}}\left(\xi^{0}\right)=0
$$

Particularly,

$$
\frac{\partial w_{\lambda_{0}}}{\partial t}\left(\xi^{0}\right)=\lim _{\delta_{k} \rightarrow 0} \frac{w_{\lambda_{k}}\left(\xi^{k}\right)}{\delta_{k}}=0 .
$$

Applying (4.9), we have

$$
\lim _{\delta_{k} \rightarrow 0} \frac{1}{\delta_{k}}\left(\left(-\Delta_{\mathbb{H}}\right)_{p}^{s} u_{\lambda_{k}}\left(\xi^{k}\right)-\left(-\Delta_{\mathbb{H}}\right)_{p}^{s} u\left(\xi^{k}\right)\right)=\lim _{\delta_{k} \rightarrow 0} \frac{1}{\delta_{k}} f^{\prime}\left(\varsigma_{\lambda_{k}}\left(\xi^{k}\right)\right) w_{\lambda_{k}}\left(\xi^{k}\right)=0,
$$


which is a contradiction with Lemma 3.3. Therefore,

$$
w_{\lambda_{0}}(\xi) \geq 0, \quad \xi \in \Sigma_{\lambda_{0}}
$$

Similarly, we can move the plane from $+\infty$ to the left to get

$$
w_{\lambda_{0}}(\xi) \leq 0, \quad \xi \in \Sigma_{\lambda_{0}}
$$

Then (4.6) follows by combining (4.10) and (4.11). Finally, we see that $u$ must be symmetric and monotone with respect to $t$ about some point.

Next, we give the proof of Theorem 1.2.

Proof of Theorem 1.2 By condition (1.8) and $f(0)=0$, we claim

$$
u(\xi)>0 \quad \text { or } \quad u(\xi) \equiv 0 \quad \text { for any } \xi \in \mathbb{H}_{+}^{n} .
$$

In fact, suppose that the conclusion $u(\xi)>0$ is not correct, we will verify $u(\xi) \equiv 0$. The lower semicontinuity of $u$ on $\mathbb{H}_{+}^{n}$ implies that there exists $\xi^{0} \in \overline{\mathbb{H}}_{+}^{n}$ such that

$$
u\left(\xi^{0}\right)=\min _{\overline{\mathbb{H}}_{+}^{n}} u=0
$$

and then

$$
\begin{aligned}
\left(-\Delta_{\mathbb{H}}\right)_{p}^{s} u\left(\xi^{0}\right) & =C_{Q, s, p} P V \int_{\mathbb{H}^{n}} \frac{\left|u\left(\xi^{0}\right)-u(\eta)\right|^{p-2}\left(u\left(\xi^{0}\right)-u(\eta)\right)}{\left|\eta^{-1} \circ \xi^{0}\right|_{\mathbb{H}}^{Q+s p}} d \eta \\
& =C_{Q, s, p} P V \int_{\mathbb{H}_{+}^{n}} \frac{\left|u\left(\xi^{0}\right)-u(\eta)\right|^{p-2}\left(u\left(\xi^{0}\right)-u(\eta)\right)}{\left|\eta^{-1} \circ \xi^{0}\right|_{\mathbb{H}}^{Q+s p}} d \eta \\
& =C_{Q, s, p} P V \int_{\mathbb{H}_{+}^{n}} \frac{-u(\eta)|u(\eta)|^{p-2}}{\left|\eta^{-1} \circ \xi^{0}\right|_{\mathbb{H}}^{Q+s p}} d \eta \\
& =f\left(u\left(\xi^{0}\right)\right)=0 .
\end{aligned}
$$

Hence $\int_{\mathbb{H}_{+}^{n}} \frac{-u(\eta)|u(\eta)|^{p-2}}{\left|\eta^{-1} \circ \xi^{0}\right| \mathbb{H}^{Q+s p}} d \eta=0$, and then $u(\xi) \equiv 0, \xi \in \mathbb{H}_{+}^{n}$.

In the sequel, we only need to treat the case $u>0$ on $\mathbb{H}_{+}^{n}$. Let us employ the method of moving planes to $u$ along the $t$ direction and denote

$$
T_{\lambda}^{+}=\left\{\xi \in \mathbb{H}_{+}^{n} \mid t=\lambda, \lambda \in \mathbb{R}^{+}\right\}
$$

and

$$
\Sigma_{\lambda}^{+}=\left\{\xi \in \mathbb{H}_{+}^{n} \mid 0<t<\lambda\right\} .
$$

The H-reflection of $\xi=(x, y, t)$ about $T_{\lambda}^{+}$is $\xi^{\lambda}=(y, x, 2 \lambda-t)$, and let

$$
w_{\lambda}(\xi)=u_{\lambda}(\xi)-u(\xi) .
$$


If $\lambda>0$ is sufficiently small, we deduce from Lemma 3.3 with $\Omega=\Sigma_{\lambda}^{+}$and $\Sigma_{\lambda}=\Sigma_{\lambda}^{+} U$ $\left(\mathbb{H}^{n} \backslash \mathbb{H}_{+}^{n}\right)$ that on the narrow region $\Sigma_{\lambda}^{+}$,

$$
w_{\lambda}(\xi) \geq 0 .
$$

This provides the starting point of moving planes. Now we will explain that the plane $T_{\lambda}^{+}$ can be moved to the infinity so that (4.12) holds. Let

$$
\lambda_{0}=\sup \left\{\lambda>0 \mid w_{\mu}(\xi) \geq 0, \forall \xi \in \Sigma_{\lambda}^{+}, \mu \leq \lambda\right\}
$$

and we will prove

$$
\lambda_{0}=\infty
$$

In fact, if $\lambda_{0}<\infty$, then we claim that $T_{\lambda}^{+}$can be moved further to the right, that is, there exists $\sigma>0$ such that, for any $\lambda \in\left(\lambda_{0}, \lambda_{0}+\sigma\right)$,

$$
w_{\lambda}(\xi) \geq 0, \quad \xi \in \Sigma_{\lambda}^{+} .
$$

This will contradict the definition of $\lambda_{0}$, and hence (4.13) holds.

At present, we prove (4.14). If $|\xi|_{\mathbb{H}}$ is sufficiently large, then (4.14) is true by using the similar proof to (4.1) in Theorem 1.1. This implies that there exists some $R_{0}>0$ such that (4.14) holds true on $\mathbb{H}_{+}^{n} \backslash B_{\mathbb{H}}\left(0, R_{0}\right)$. Next we point out that (4.14) is also true on $B_{\mathbb{H}}\left(0, R_{0}\right)$. Noting $\lambda_{0}<\infty$ and using Lemma 3.2, we find that on $\xi \in \Sigma_{\lambda_{0}}^{+} \cap B_{\mathbb{H}}\left(0, R_{0}\right)$,

$$
w_{\lambda_{0}}(\xi)>0
$$

or

$$
w_{\lambda_{0}}(\xi) \equiv 0
$$

In the case $w_{\lambda_{0}}(\xi) \equiv 0$, we observe by the boundary conditions of $u$ that $u(\xi) \equiv 0$. On the other hand, (4.15) implies that there exists small $\delta>0$ such that

$$
w_{\lambda_{0}}(\xi) \geq c>0, \quad \xi \in \overline{\Sigma_{\lambda_{0}-\delta}^{+} \cap B_{\mathbb{H}}\left(0, R_{0}\right)} .
$$

Since $w_{\lambda}$ relies continuously on $\lambda$, there exists $\sigma>\varepsilon>0$ such that

$$
w_{\lambda_{0}+\varepsilon}(\xi) \geq 0, \quad \xi \in \overline{\Sigma_{\lambda_{0}-\delta}^{+} \cap B_{\mathbb{H}}\left(0, R_{0}\right)}
$$

Since $\left(\Sigma_{\lambda_{0}+\varepsilon}^{+} \backslash \Sigma_{\lambda_{0}-\delta}^{+}\right) \cap B_{\mathbb{H}}\left(0, R_{0}\right)$ is a narrow region, we have by Lemma 3.3

$$
w_{\lambda_{0}+\varepsilon}(\xi) \geq 0, \quad \xi \in \overline{\Sigma_{\lambda_{0}+\varepsilon}^{+} \cap B_{\mathbb{H}}\left(0, R_{0}\right)}
$$

and therefore (4.14) is proved. 
Using (4.13) and Lemma 3.2 once again, it follows that, for any $\xi \in \Sigma_{\lambda}^{+}$(here $\left.0 \leq \lambda \leq \infty\right)$,

$$
w_{\lambda}(\xi)>0,
$$

or

$$
w_{\lambda}(\xi) \equiv 0 .
$$

For (4.19), we can use the boundary condition of $u$ to obtain $u(\xi) \equiv 0$. In addition, it follows from (4.18) that $u(\xi)$ is strictly increasing, which contradicts the boundary condition of $u$ and (1.8).

Funding

This work is supported by the National Natural Science Foundation of China (11701162); National Science Foundation of Shandong Province of China (ZR2019MA067); Research Fund for the Doctoral Program of Hubei University of Economics (XJ16BS28); and Guangxi Natural Science Foundation (2017GXNSFBA198130).

Availability of data and materials

Not applicable.

Ethics approval and consent to participate

Not applicable.

Competing interests

The authors declare that they have no competing interests.

Consent for publication

Not applicable.

Authors' contributions

All authors read and approved the final manuscript.

\section{Author details}

'School of Mathematics and Statistics and Zhumadian Academy of Industry Innovation and Development, Huanghuai University, Zhumadian, Henan 463000, China. ${ }^{2}$ School of Mathematical Sciences, Qufu Normal University, Qufu, Shandong 273165, China.

\section{Publisher's Note}

Springer Nature remains neutral with regard to jurisdictional claims in published maps and institutional affiliations.

Received: 3 March 2020 Accepted: 13 July 2020 Published online: 22 July 2020

\section{References}

1. Birindelli, I., Prajapat, J.: Nonlinear Liouville theorems in the Heisenberg group via the moving plane method. Commun. Partial Differ. Equ. 24(9-10), 1875-1890 (1999)

2. Bony, J.M.: Principe du Maximum, Inegalite de Harnack et unicite du probleme de Cauchy pour les operateurs ellipitiques degeneres. Ann. Inst. Fourier (Grenoble) 19, 277-304 (1969)

3. Brändle, C., Colorado, E., de Pablo, A., Sánchez, U.: A concave convex elliptic problem involving the fractional Laplacian. Proc. R. Soc. Edinb. 143, 39-71 (2013)

4. Caffarelli, L., Silvestre, L.: An extension problem related to the fractional Laplacian. Commun. Partial Differ. Equ. 2, $1245-1260(2007)$

5. Chen, W., Li, C.: Methods on Nonlinear Elliptic Equations. AIMS Book Series, vol. 4 (2010)

6. Chen, W., Li, C.: Maximum principles for the fractional p-Laplacian and symmetry of solutions. Adv. Math. 335, 735-758 (2018)

7. Chen, W., Li, C., Li, Y.: A direct method of moving planes for the fractional Laplacian. Adv. Math. 308, 404-437 (2016)

8. Cinti, E., Tan, J.: A nonlinear Liouville theorem for fractional equations in the Heisenberg group. J. Math. Anal. Appl. 433, 434-454 (2016)

9. Cowling, M., Haagerup, U.: Completely bounded multipliers of the Fourier algebra of a simple Lie group of real rank one. Invent. Math. 96, 507-549 (1989)

10. Dou, J., Niu, P.: Nonexistence of weak solutions for $p$-sub-Laplacian inequalities on the Heisenberg group. J. Jilin Univ. Sci. Ed. 44, 163-169 (2006)

11. Dou, J., Niu, P., Yuan, Z.: A Hardy inequality with remainder terms in the Heisenberg group and the weighted eigenvalue problem. J. Inequal. Appl. 2007(1), 1 (2007)

12. Ferrari, F., Franchi, B.: Harnack inequality for fractional Laplacians in Carnot groups. Math. Z. 279, 435-458 (2015) 
13. Folland, G.B., Stein, E.M.: Estimates for the $\bar{\partial}_{b}$ complex and analysis on the Heisenberg group. Commun. Pure Appl. Math. 27(4), 429-522 (1974)

14. Frank, R., Gonzalez, M., Monticelli, D., Tan, J.: An extension problem for the CR fractional Laplacian. Adv. Math. 270, 97-137 (2015)

15. Garofalo, N., Lanconelli, E.: Existence and nonexistence results for semilinear equations on the Heisenberg group. Indiana Univ. Math. J. 41, 71-98 (1992)

16. Hörmander, L.: Hypoelliptic second order differential equations. Acta Math. 119, 147-171 (1967)

17. Jerison, D.: Boundary regularity in the Dirichlet problem for $\square_{b}$ on CR manifolds. Commun. Pure Appl. Math. 36(2), 143-181 (1983)

18. Jerison, D.S., Lee, J.M.: The Yamabe problem on CR manifolds. J. Differ. Geom. 25, 167-197 (1987)

19. Li, Y., Ni, W.M.: Radial symmetry of positive solutions of nonlinear elliptic equations in $R^{n}$. Commun. Partial Differ. Equ. $18,1043-1054$ (1993)

20. Liu, H., Niu, P.: Maximum principles of nonhomogeneous subelliptic p-Laplace equations and applications. J. Partial Differ. Equ. 4, 289-303 (2006)

21. Liu, H., Niu, P.: Growth estimates to subelliptic $p$-Laplace equations on Heisenberg group. J. Grad. Sch. Chin. Acad. Sci. 24(1), 18-24 (2007)

22. Roncal, L., Thangavelu, S.: Hardy's inequality for fractional powers of the subLaplacian on the Heisenberg group. Adv. Math. 302, 106-158 (2016)

23. Wang, X., Cui, X., Niu, P.: A Liouville theorem for the semilinear fractional CR covariant equation on the Heisenberg group. Complex Var. Elliptic Equ. 64(8), 1325-1344 (2019)

24. Wang, X., Niu, P., Cui, X.: A Liouville type theorem to an extension problem relating to the Heisenberg group. Commun. Pure Appl. Anal. 17(6), 2379-2394 (2018)

25. Wei, N., Niu, P., Liu, H.: Dirichlet eigenvalue ratios for the p-sub-Laplacian in the Carnot group. J. Partial Differ. Equ. 22(1), 1-10 (2009)

26. Yuan, Z., Niu, P.: A Hopf type principle and a strong maximum principle for the p-sub-Laplacian on the group of Heisenberg type. J. Math. Res. Expo. 27(3), 605-612 (2007)

27. Yuan, Z., Niu, P.: Some Liouville type theorems for the $p$-sub-Laplacian on the group of Heisenberg type. J. Partial Differ. Equ. 3, 193-207 (2008)

\section{Submit your manuscript to a SpringerOpen ${ }^{0}$ journal and benefit from:}

- Convenient online submission

- Rigorous peer review

- Open access: articles freely available online

- High visibility within the field

- Retaining the copyright to your article

Submit your next manuscript at $\boldsymbol{~ s p r i n g e r o p e n . c o m ~}$ 